\title{
Horizontal locomotion of a vertically flapping oblate spheroid
}

\author{
Jian Deng $^{1}$ and C. P. Caulfield ${ }^{2,3} \dagger$ \\ ${ }^{1}$ Department of Mechanics, Zhejiang University, Hangzhou 310027, P R China \\ ${ }^{2}$ BP Institute, University of Cambridge, Madingley Road, Cambridge CB3 0EZ, UK \\ ${ }^{3}$ Department of Applied Mathematics and Theoretical Physics, University of Cambridge, \\ Centre for Mathematical Sciences, Wilberforce Road, Cambridge CB3 0WA, UK
}

(Received 8 January 2018..; revised ..; accepted ... - To be entered by editorial office)

We consider the self-induced motions of three-dimensional oblate spheroids of density $\rho_{s}$ with varying aspect ratios $A R=b / c \leqslant 1$, where $b$ and $c$ are the spheroids' centrepole radius and centre-equator radius respectively. Vertical motion is imposed on the spheroids such that $y_{s}(t)=A \sin (2 \pi f t)$ in a fluid of density $\rho$ and kinematic viscosity $\nu$. As in strictly two-dimensional flows, above a critical value $R e_{C}$ of the flapping Reynolds number $R e_{A}=2 A f c / \nu$ the spheroid ultimately propels itself horizontally as a result of fluid-body interactions. For $R e_{A}$ sufficiently above $R e_{C}$, the spheroid rapidly settles into a terminal state of constant, unidirectional velocity, consistent with the prediction of Deng et al. (2016) that, at sufficiently high $R e_{A}$, such oscillating spheroids manifest $m=1$ asymmetric flow, with characteristic vortical structures conducive to providing unidirectional thrust if the spheroid is free to move horizontally. The speed $U$ of propagation increases linearly with the flapping frequency, resulting in a constant Strouhal number $S t(A R)=2 A f / U$, characterising the locomotive performance of the oblate spheroid, somewhat larger than the equivalent $S t$ for two-dimensional spheroids, demonstrating that the three-dimensional flow is less efficient at driving locomotion. $S t$ decreases with increasing aspect ratio for both two-dimensional and three-dimensional flows, although the relative disparity (and hence relative inefficiency of three-dimensional motion) decreases. For flows with $R e_{A} \gtrsim R e_{C}$, we observe two distinct types of inherently threedimensional motion for different aspect ratios. The first, associated with a disk of aspect ratio $A R=0.1$ at $R e_{A}=45$, consists of a 'stair-step' trajectory. This trajectory can be understood through consideration of relatively high azimuthal wavenumber instabilities of interacting vortex rings, characterised by in-phase vortical structures above and below an oscillating spheroid, recently calculated using Floquet analysis by Deng et al. (2016). Such 'in-phase' instabilities arise in a relatively narrow band of $R e_{A} \gtrsim R e_{C}$, which band shifts to higher Reynolds number as the aspect ratio increases. (Indeed, for horizontally fixed spheroids with aspect ratio $A R=0.2$, Floquet analysis actually predicts stability at $R e_{A}=45$.) For such a spheroid $\left(A R=0.2, R e_{A}=45\right.$, with sufficiently small mass ratio $m_{s} / m_{f}=\rho_{s} V_{s} /\left(\rho V_{s}\right)$ where $V_{s}$ is the volume of the spheroid) which is free to move horizontally the second type of three-dimensional motion is observed, initially taking the form of a 'snaking' trajectory with, long quasi-periodic sweeping oscillations before locking into an approximately elliptical 'orbit', apparently manifesting a three-dimensional generalization of the $Q P_{H}$ quasi-periodic symmetry breaking discussed for sufficiently high aspect ratio two-dimensional elliptical foils in Deng \& Caulfield (2016).

$\dagger$ Email address for correspondence: c.p.caulfield@bpi.cam.ac.uk 


\section{Introduction}

Flapping wings or fins are commonly used by flying or swimming animals for locomotion through a fluid (Childress 1981; Alexander 1993). This strategy has also been implemented to design underwater vehicles using flapping propellers for propulsion (Fish et al. 2003; Techet et al. 2004). To understand such thrust generation mechanisms, there have been many experimental and numerical studies of the flow dynamics of flapping foils (Triantafyllou et al. 1993, 2004; Lewin \& Haj-Hariri 2003). For example, recently, Klotsa et al. (2015) put forward a conceptual design for a 'two-sphere swimmer'. They studied the propulsion of a neutrally buoyant swimmer consisting of a pair of spheres attached by a spring, immersed in a vibrating fluid. They reported that the vibration of the fluid induces relative motion of the spheres which, for sufficiently large amplitudes, can lead to motion of the centre of mass of the two spheres.

This type of self-propelled 'robot' has actually become an important technological and medical challenge that brings together elements of physics, chemistry, biology, engineering, and fluid mechanics (Dreyfus et al. 2005; Bar-Cohen 2006; Williams et al. 2014). Most of the artificial microscopic 'robots', as well as many of their naturally occurring counterparts, for example insects and aquatic invertebrates, operate at intermediate Reynolds numbers (1-100) (Childress \& Dudley 2004), where nonlinearity is expected to be important, yet the flow is not expected to undergo the transition to turbulence. However, from the perspective of fluid mechanics, how time-reversal symmetry is broken by nonlinearities as the Reynolds number increases is still not clearly established, particularly for (realistic) three-dimensional flows. Therefore, we believe that a further study on self-propulsion is meaningful to understand physical mechanisms potentially relevant to both animal and artificial 'robot' locomotion.

Vandenberghe et al. (2004) proposed a self-propelled model flow, considering a twodimensional plate of length $2 c$ which was free to move horizontally as a result of a prescribed oscillatory vertical motion $y(t)=A \sin (2 \pi f t)$. They showed that the flow symmetry about the midpoint of the vertically oscillating plate breaks at some critical amplitude, leading to the plate 'flying' forwards. Above a critical value $R e_{C}$ of the flapping Reynolds number $R e_{A}$, they observed an approximately linear increase in the forward speed $U$ with flapping frequency, implying a constant value of the Strouhal number $S t \simeq 0.26$ as $R e_{A}$ increases, where $R e_{A}$ and $S t$ are defined as

$$
R e_{A}=\frac{2 A f c}{\nu} ; S t=\frac{2 f A}{U},
$$

where $\nu$ is the kinematic viscosity of the fluid. This result is consistent (though perhaps coincidental) with our understanding of the flapping locomotion of animals, as Triantafyllou et al. (1993) noted that the range $0.25<S t<0.35$ may be considered to be optimal for thrust production. Using numerical simulations, Alben \& Shelley (2005) showed how simple imposed oscillations of two-dimensional ellipses of varying aspect ratio can lead to forward locomotion. They identified that there are two different identifiable stages as the oscillating body undergoes the transition to forward motion. First, they showed that the fluid flow loses symmetry through a linear instability. Second, nonlinear fluid-solid interactions between the body and previously shed vortices (associated with previous oscillation cycles) eventually 'push' the body into forward locomotion. Alben \& Shelley (2005) found that the critical value for the onset of such locomotion $R e_{C}$ is independent of density ratio $\rho_{s} / \rho$, where $\rho_{s}$ is the density of the solid body, implying that the primary instability is that of the fluid flow alone and not of the coupled body-fluid system.

Subsequently, the dynamics of a two-dimensional flapping body in a viscous fluid has been extensively investigated (Vandenberghe et al. 2006; Lu \& Liao 2006; Zhang et al. 
2009). Although many new phenomena have been identified, most previous research has been based on the hypothesis that it is appropriate to consider purely two-dimensional flow. For example, we have recently shown (see Deng \& Caulfield (2016)) that the onset of symmetry breaking around elliptical foils of varying aspect ratios which are free to move horizontally has many points of similarity with the onset of symmetry breaking around fixed cylinders (Tatsuno \& Bearman 1990; Elston et al. 2001). In particular, we showed that the character of the symmetry breaking which occurs depends on the values of two natural nondimensional parameters: the nondimensional amplitude or KeuleganCarpenter number $K C$ and the nondimensional frequency or Stokes number $\beta$ defined, conventionally using the diameter $2 c$ of the foil, as

$$
K C=\frac{2 \pi A}{2 c} ; \beta=\frac{f(2 c)^{2}}{\nu} ; \operatorname{Re}_{A}=\frac{\beta K C}{2 \pi} .
$$

For sufficiently small values of $\beta$, the onset of asymmetry around both fixed and freeto-fly foils is 'synchronous', in that the observed horizontal force is completely dominated by a frequency double that of the primary oscillation. Conversely, for larger values of $\beta$ and also larger aspect ratios, the asymmetry around both fixed and flying foils is 'quasiperiodic', in that the horizontal force exhibits two distinct frequencies, leading typically to a long period beating in the horizontal motion of the foil. We labelled this particular type of symmetry breaking as ' $Q P_{H}$ ' symmetry breaking. However, for flying two-dimensional foils with small or 'low' aspect ratios $(A R=0.1)$, we observed that the quasi-periodic asymmetry (which we labelled as ' $Q P_{L}$ ' symmetry breaking) exhibited by fixed foils was actually suppressed, with the symmetry-breaking for flying foils being synchronous in character. We also observed that the loss of symmetry around relatively higher aspect ratio foils at relatively high $\beta$ was encouraged by the foil being free to move horizontally, in that it occurred at smaller $R e_{A}$ than for fixed foils.

In this paper, our central focus is to investigate whether intuition gained from purely two-dimensional studies is relevant to the flapping-induced locomotion of more realistic three-dimensional bodies. Of particular motivation is the (perhaps unsurprising) observation that three-dimensional oscillating bodies are prone to symmetry-breaking instabilities. Focussing on oblate spheroids with aspect ratio $A R=0.1$ and using Floquet stability analyses, in Deng et al. (2016) we demonstrated that there exists a rich variety of relatively high azimuthal wavenumber instabilities for an intermediate range of $K C, \beta$ pairs between stable symmetric flow and (at sufficiently high $K C$ ) asymmetric flow with $m=1$ structure. These instabilities can be classified into two branches, 'I' and ' $\mathrm{O}$ ', with vortical structures either in or out of phase above and below the oscillating spheroid. Branch ' $\mathrm{I}$ ' occurs for smaller $K C$ (and typically at lower wavenumber $m$ ) for given $\beta$, and thus branch ' $\mathrm{I}$ ' is the first symmetry-breaking instability to occur as the amplitude of the oscillation increases. Finite amplitude manifestations of these instabilities have been observed experimentally (Deng et al. 2017) once again only for the relatively small aspect ratio $A R=0.1$, and so it is naturally of interest to investigate whether these instabilities, particularly for such smaller aspect ratio spheroids, play any role in any symmetry-breaking leading (at finite amplitude) to locomotion. In such an investigation it is always important to remember two aspects of the evidence from our two-dimensional studies. First, these studies suggest that linear stability properties are strongly dependent on aspect ratio, and so extrapolating results from one aspect ratio to another should be treated with caution. Secondly, these studies also suggest that, close to the onset of symmetry-breaking, the behaviour of horizontally-fixed and freely-moving bodies can be different. In particular, both stability thresholds derived from studies of horizontally-fixed bodies and the existence of quasi-periodic finite amplitude oscillatory 
states may well not carry over quantitatively to bodies which are free to move horizontally. As we discuss in more detail below, these two aspects appear to remain significant in three-dimensional flows.

For simplicity, and also to connect with our recent investigations both of symmetrybreaking around two-dimensional elliptical foils and of high-wavenumber instability around (horizontally fixed) oblate spheroids, we model the flapping body as just such an oblate spheroid, with varying aspect ratio, subject to a specified sinusoidal vertical oscillation and in general free to move horizontally in response to the fluid forces acting upon it. We restrict attention to a fixed, sufficiently large value of scaled frequency or Stokes number $(\beta=500$ as defined in (1.2)) for two related reasons. First, this parameter value allows us to investigate whether the onset of asymmetry remains quasi-periodic in character for three-dimensional bodies with higher aspect ratios (i.e. $A R>0.1$ ), with quasi-periodicity conversely continuing to be suppressed for bodies with sufficiently small aspect ratios $(A R=0.1)$ as observed in two dimensions in Deng \& Caulfield (2016). Second, we can also assess whether the branch ' $\mathrm{I}$ ' or branch ' $\mathrm{O}$ ' high azimuthal wavenumber instabilities play any role in the transition to locomotion. For this choice of $\beta$, there is a nontrivial range of scaled amplitudes $K C$ where branch ' $\mathrm{I}$ ' and branch ' $\mathrm{O}$ ' instabilities occur for horizontally fixed three-dimensional oblate spheroids before the flow eventually manifests a simpler $m=1$ asymmetry, with an associated flow structure similar to the flow characteristic of locomotion for flapping freely-moving bodies.

Specifically, our aims are to reveal two fundamental aspects of the three-dimensional flow dynamics and compare and contrast them with the equivalent two-dimensional behaviour. First, we show that there still exists an attracting dynamical state in this threedimensional flow, such that the body maintains a stable unidirectional locomotion at sufficiently high $R e_{A}$, and we identify the central physical processes associated with this flow state, in particular that it is somewhat less efficient than the equivalent two-dimensional flow, and show that this relative inefficiency becomes less significant as aspect ratio increases. Second, we show that sufficiently close to yet above the critical value $R e_{C}$ of $R e_{A}$ for locomotion, the induced, inherently three-dimensional vortical motions can lead to complex trajectories for the body. For $A R=0.1$ in a flow with $R e_{A}=45$, the trajectory can be related directly to a modulated version of the dominant branch ' $I$ ' $m=4$ linear instability, strongly suggesting that the suppression of quasi-periodicity and the dominance at onset of synchronous asymmetry observed in two-dimensional flows also occurs for three-dimensional small-aspect-ratio bodies. Conversely, for $A R=0.2$, the flow with $R e_{A}=45$ induced by a horizontally-fixed spheroid is not prone to the highly organised branch 'I' instabilities, illustrating that the stability properties are indeed strongly dependent on the aspect ratio of the oscillating body, but rather undergoes the transition to asymmetry via a three-dimensional generalisation of the $Q P_{H}$-type quasi-periodicity also previously observed in two dimensions. To address these aims, the rest of the paper is organised as follows. In section 2, we briefly review our numerical methods both for direct simulation and Floquet stability analysis, and describe the specific parameters for our simulations. In section 3, we present our results, particularly placing them into the context of our previous two-dimensional and Floquet instability studies, described in Deng et al. (2016) and Deng et al. (2017). We then draw our conclusions in section 4. 


\section{Numerical methods}

\subsection{Flow parameters}

We consider an oblate spheroid of uniform density $\rho_{s}$ and volume $V_{s}$, which is formed by rotation about the minor vertical $y$-axis of an ellipse with semi-major radius $c$ and semiminor radius $b$. We define the aspect ratio or oblateness as $\mathrm{AR}=b / c \leqslant 1$, and consider the motion of spheroids with $A R=0.1,0.2,0.3$ and 0.4 . We impose a fixed vertical motion of $y_{s}=A \sin (2 \pi f t)$ on the spheroid, which is in general free to move in the horizontal plane in a fluid of density $\rho$ and kinematic viscosity $\nu$. Therefore, there are two appropriate Reynolds numbers for this flow, the flapping Reynolds number $R e_{A}$ as defined in (1.1), and the locomotion Reynolds number $R e_{U}$, defined as

$$
R e_{U}=\frac{2 U c}{\nu}, U=|\mathbf{u}|,
$$

where $\mathbf{u}$ is the asymptotic steady horizontal velocity vector for the flapping body. We vary $R e_{A} \in[27,99]$, and $R e_{U}$ emerges as a property of the ensuing motion. As already noted, we fix the scaled frequency or Stokes number $\beta=500$, from (1.2), so variation of $R e_{A}$ is equivalent to variation in the scaled amplitude or Keulegan-Carpenter number $K C$. Also, the Strouhal number $S t$ as defined in (1.1) can then be identified as $S t=$ $2 R e_{A} / R e_{U}$, consistently with previous definitions used in the characterisation of the wake dynamics of a flapping foil (Taylor et al. 2003; Triantafyllou et al. 1993). For comparison, we also conduct a range of two-dimensional simulations of flapping elliptical foils with $A R \in[0.1,0.7]$ and $R e_{A} \in[9,135]$.

\subsection{Direct numerical simulation}

We use the open source code OpenFOAM (Jasak 1996). The time-dependent NavierStokes equations are solved using the finite volume method, assuming incompressibility, and with no turbulence closure. The mass and momentum equations are solved on a moving grid domain using the Arbitrary Lagrangian Eulerian (ALE) formulation (see (Ferziger \& Peric 2002)). The integral form of the governing (conservation) equations defined in an arbitrary moving volume $V$ bounded by a closed surface $S$ is

$$
\frac{d}{d t} \int_{V} \rho \mathbf{U} d V+\oint_{S} \mathbf{d} \mathbf{s} \cdot \rho\left(\mathbf{U}-\mathbf{U}_{b}\right) \mathbf{U}=\oint_{S} \mathbf{d} \mathbf{s} \cdot(-p \mathbf{I}+\rho \nu \nabla \mathbf{U}),
$$

where $\mathbf{U}$ is the fluid velocity, $\mathbf{U}_{b}$ is the boundary velocity of a finite volume, $\rho$ is the fluid density, and $p$ is the pressure. As the volume $V$ is no longer fixed in space, its motion is captured by the motion of its bounding surface $S$ at the boundary velocity $\mathbf{U}_{b}$. For the details of the discretisation and implementation of boundary conditions, as well as the transformation of the underlying partial differential equations into corresponding systems of algebraic equations, see Ferziger \& Peric (2002).

The space discretizations are second-order upwind for the convection terms and central differences for the Laplacian terms, respectively. The time discretization is first-order implicit Euler. The pressure-velocity coupling in incompressible flow simulations is obtained using the PISO scheme (Ferziger \& Peric 2002). Numerical accuracy is set to double-precision and the initial conditions are chosen to be uniform. We set the boundary condition on the spheroid to be moving-wall, with no flux normal to the wall. At the outer boundary of the computational domain (a sphere with a radius of 40c) we set the normal components of the velocity gradients to be zero, and the pressure to be zero.

We have found that at least 2000 time steps are required to ensure that the unsteadiness caused by the oscillation is well-resolved. To determine the required level of spatial 
resolution, we simulate a purely oscillating circular two dimension foil at three different mesh resolutions: a coarse mesh (31250 cells); a medium mesh (50236 cells); and a fine mesh $(86936$ cells). We find that both the medium mesh and fine mesh resolution provide satisfactory accuracy in space, and so we generate a three-dimensional mesh through simple rotation about the vertical axis of the medium mesh with axisymmetric horizontal resolution, leading to a three-dimensional mesh with about 1.5 million cells. As noted below, when appropriate we also check the robustness of our new (and perhaps surprising) results to numerical resolution by repeating simulations on an appreciably finer mesh with approximately 3.5 million cells. As already noted, the vertical motion of the spheroid is specified, while its horizontal motion is determined by the horizontal fluid force using Newton's second law:

$$
m_{s} \frac{d^{2}\left(x_{b}, z_{b}\right)}{d t^{2}}=\left(F_{x}, F_{z}\right),
$$

where $\left(\mathbf{x}_{b}=\left(x_{b}, z_{b}\right)\right)$ is the horizontal location vector of the spheroid in the $(x, z)$ plane, $\mathbf{F}_{B}=\left(F_{x}, F_{z}\right)$ is the horizontal fluid force vector acting on the spheroid, $m_{s}$ is the mass of the spheroid given by $m_{s}=\rho_{s} V_{s}$, with $\rho_{s}$ the density of the spheroid and $V$ the body volume. The fluid mass with equivalent volume is denoted as $m_{f}=\rho V_{s}$. In most simulations, if not specifically noted, we keep the mass ratio at $m_{s} / m_{f}=10$. We solve (2.3) using a fourth-order Runge-Kutta time-stepping scheme.

In the current study, all lengths are non-dimensionalized with $2 c$, all densities are non-dimensionalized with $\rho$, and all times are non-dimensionalized with the viscous time scale $(2 c)^{2} / \nu$. In the following discussion, all forces are scaled by $10^{4} \rho \nu^{2} / 2$, velocities are scaled by $10^{2} \nu /(2 c)$, and the displacements are scaled by $2 c$.

\subsection{Floquet stability analysis}

As discussed in more detail in Deng et al. (2016), the linear stability of a (horizontally fixed) oscillating spheroid can be studied using Floquet analysis, since the underlying flow has a natural periodicity. In brief, we decompose the flow field around the oscillating spheroid into an axisymmetric base flow and a small amplitude perturbation, $(\mathbf{u}, p)=$ $(\mathbf{U}, P)+\left(\mathbf{u}^{\prime}, p^{\prime}\right)$, which perturbation satisfies

$$
\frac{\partial \mathbf{u}^{\prime}}{\partial t}=-\mathbf{U} \cdot \nabla \mathbf{u}^{\prime}-\mathbf{u}^{\prime} \cdot \nabla \mathbf{U}-\nabla p^{\prime}+\nu \nabla^{2} \mathbf{u}^{\prime} ; \boldsymbol{\nabla} \cdot \mathbf{u}^{\prime}=0 .
$$

We use a spectral-element method to calculate both the axisymmetric base flow and the instabilities (Karniadakis \& Sherwin 2013). Any perturbation at time $t=T$ can be decomposed as the sum of azimuthal modes with wavenumber $m$ :

$$
\mathbf{u}^{\prime}(r, \theta, z, t)=\sum_{m=0}^{+\infty} \mathbf{u}^{(m)}(r, z, t) e^{i m \theta},
$$

We then define a ( $T$-periodic) operator $\mathbf{A}$, which evolves a perturbation from $t=0$ to $t=T=2 \pi / f_{0}$ by integrating (2.4) forward in time, and so:

$$
\mathbf{u}^{\prime}(T)=\mathbf{A}(T) \mathbf{u}^{\prime}(0) .
$$

The Floquet modes and their multipliers, labelled $\mu$, can be obtained by calculating the eigenvectors and eigenvalues of $\mathbf{A}$, through applying an Arnoldi method to a Krylov subspace constructed by iteratively integrating (2.4). For the flows considered here, these eigenvalues are all real, and the dominant (largest) one, denoted as $\mu_{\max }$, corresponds to the least stable mode. If $\mu_{\max }>1$, this mode is unstable and will exhibit perturbation 

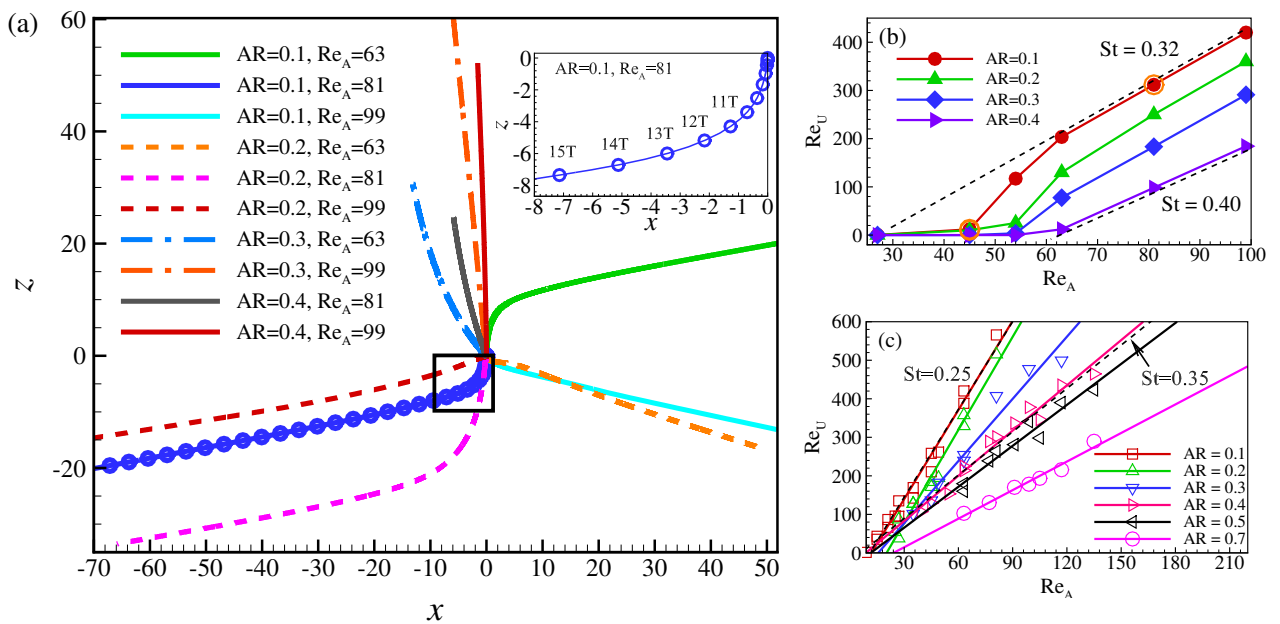

FiguRE 1. (a) Trajectories projected to the horizontal plane for unidirectional locomotion for a range of spheroids with different aspect ratio and $R e_{A}$, as denoted by the line type. The locations of the spheroid with $A R=0.1$ and $R e_{A}=81$ at the various times shown in figure 2 are marked with symbols in the magnified inset to highlight the first 15 cycles. Variation of the locomotion Reynolds number $R e_{U}$ with flapping Reynolds number $R e_{A}$ for: (b) flapping oblate spheroids; and (c) two-dimensional flapping foils. Various aspect ratios are marked with different symbols and line types, while the three cases highlighted in figures 2-8 are circled in (b). For comparison, the previous range of dependencies identified by Taylor et al. (2003) (i.e. $0.25<S t<0.35$ are marked with two dashed lines in (c).

energy growth, with growth rate given by the equivalent Floquet exponent $\sigma_{\max }$ defined implicitly by $\mu_{\max }=\exp \left(\sigma_{\max } T\right)$.

\section{Results}

\subsection{Unidirectional locomotion}

For a two-dimensional flapping elliptical foil which is not moving horizontally initially, at sufficiently large flapping Reynolds number $R e_{A}$, as defined in (1.1), unidirectional locomotion emerges as an attracting state. (Alben \& Shelley 2005). We find that a flapping oblate spheroid is also ultimately attracted to unidirectional horizontal locomotion, but due to three-dimensionality, the transition is (unsurprisingly) appreciably more complex. In figure 1(a), we present typical trajectories for various cases ultimately attracted to unidirectional locomotion away from the initial location of the foil. Some cases exhibit turning manoeuvres before settling down to their final direction of propagation. Due to inevitable numerical perturbations, we believe that there is no deterministic pattern to the selection of the eventual direction of propagation, although it is clear that unidirectional motion is both a strongly attracting and stable state for these particular choices of aspect ratio and $R e_{A}$.

In figure 1(b), we plot the variation of locomotion Reynolds number $R e_{U}$ with the flapping Reynolds number $R e_{A}$ for flapping oblate spheroids with various aspect ratios. For comparison, in figure 1(c), we plot the variation of $R e_{U}$ with $R e_{A}$ for two-dimensional flapping foils with various aspect ratios. For the two-dimensional flapping foils with $A R=$ $0.1,0.2$ and $0.3, S t=0.25,0.26$ and 0.32 respectively, which fall in the range $0.25<S t<$ 0.35 , reported by Taylor et al. (2003) to be optimal for flying and swimming organisms performing thrust motion. It is important to appreciate that this consistency may be a 


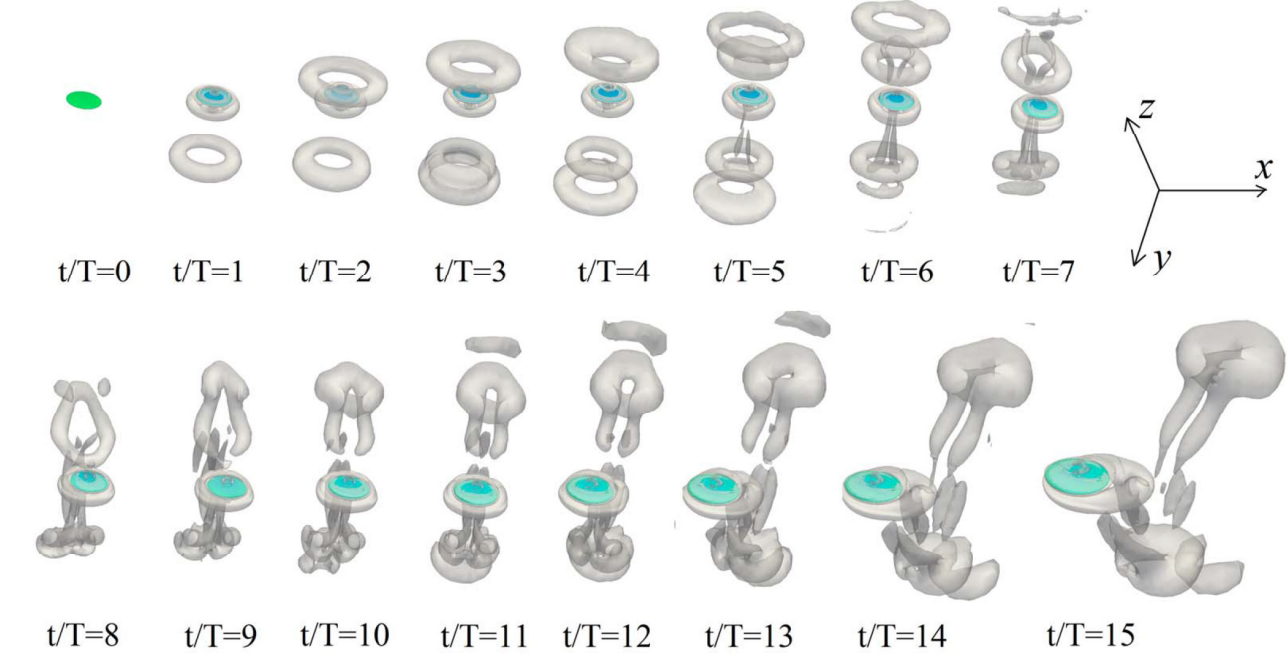

FiguRE 2. Vortex topologies around the flapping oblate spheroid at $R e_{A}=81$ and $A R=0.1$, visualized using $Q=2$, as defined in (3.1). Images demonstrate the initial flow dynamics leading to unidirectional locomotion. The disk is coloured with the surface pressure distribution. A movie is also available as supplementary material.

coincidence, as the specific characteristics of the flows around these idealised bodies and around organisms are undoubtedly different. Nevertheless, it is undoubtedly of interest to investigate whether these particular values of $S t$ are subject to a fundamental physical bound of some kind associated with the streamlined shape of such low aspect ratio bodies. Indeed, for larger aspect ratios, locomotion through flapping motion is greatly reduced, with $S t=0.52,0.7$ and 0.81 for $A R=0.4,0.5$ and 0.7 respectively.

For the flapping oblate spheroids, $S t=0.32$ and 0.40 for $A R=0.1$ and 0.4 respectively, implying a performance degradation as $A R$ increases, although this effect is less significant than in the strictly two-dimensional flows. Therefore, the locomotion of threedimensional spheroids is less efficient than the equivalent two-dimensional foils with the same aspect ratio, in that $S t$ is larger, and hence the ultimate locomotion speed $U$ is smaller for a given amplitude of oscillation $A$. This relative inefficiency is approximately $30 \%$ for bodies with $A R=0.1$, and this relative inefficiency decreases with increasing aspect ratio, dropping to approximately $10 \%$ for bodies with $A R=0.4$. The fact that the efficiency of an oblate spheroid is less sensitive to the aspect ratio than two-dimensional elliptical foils is perhaps unsurprising, as the flow around an oblate spheroid is not so strongly affected by increases in aspect ratio. Specifically, the oscillating oblate spheroid still sheds toroidal vortex rings during the oscillation, and the instability and nonlinear interaction (intimately related to three-dimensional vortex stretching) of these rings leads to a largely similar locomotion of the spheroid over a range of aspect ratios. Conversely, the coupling (leading to locomotion) of the vortices shed from two-dimensional elliptical foils is more strongly affected by increases in aspect ratio.

We have not focussed on identifying precisely the critical values $\operatorname{Re}_{C}(A R)$ of $\operatorname{Re}_{A}$ at which the spheroid starts to move horizontally, Nevertheless, it appears that these critical values fall in the range $R e_{C} \simeq 45-65$, and increase with aspect ratio, which is consistent with the previously considered two-dimensional flow(Alben \& Shelley 2005), although three-dimensionality in the flow delays the onset of locomotion to larger flapping amplitudes, and hence larger $\operatorname{Re}_{A}$ (since $\beta$ is fixed). To understand the underlying physical mechanisms leading to unidirectional propagation, we visualise the evolution of vorticity 

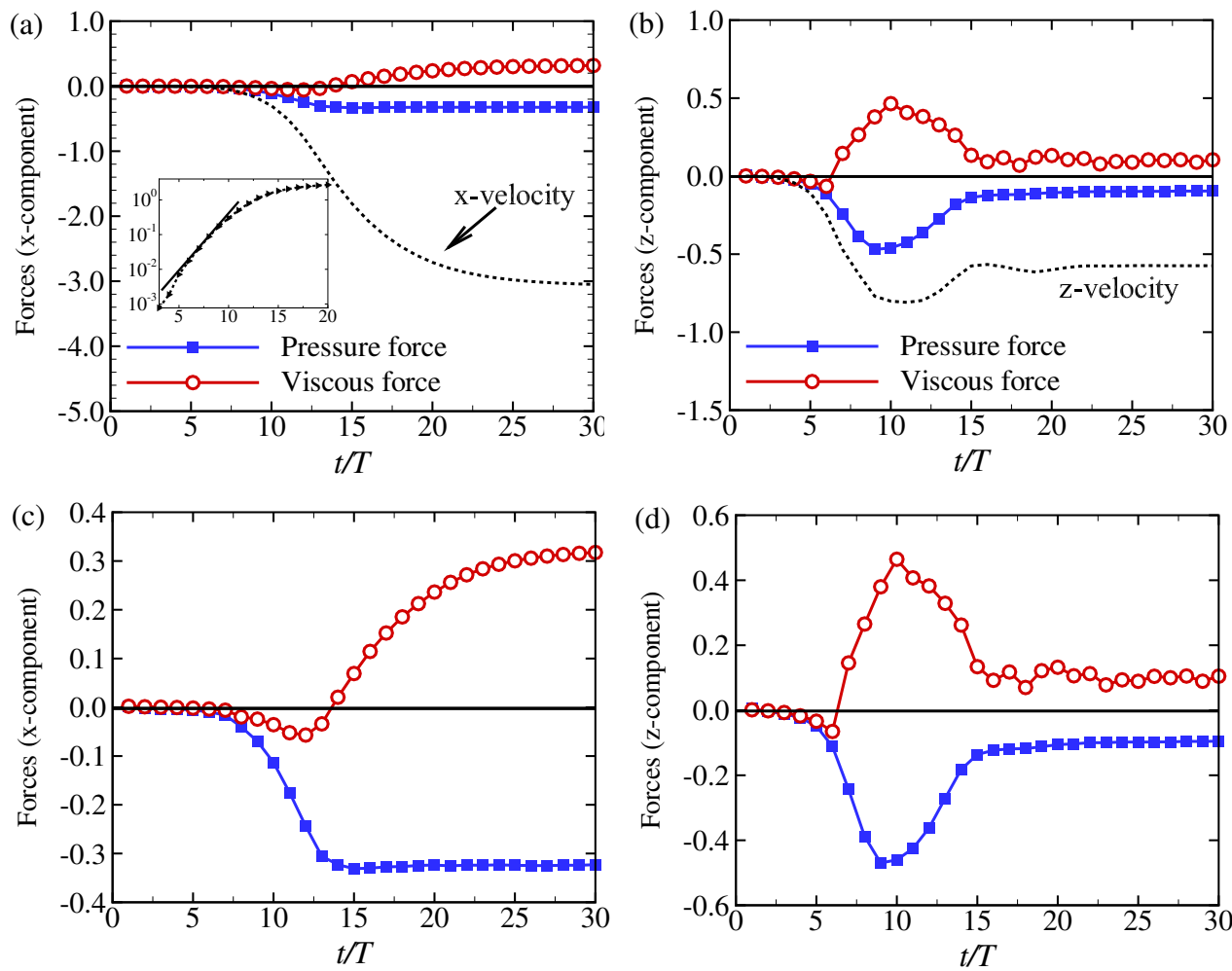

FiguRE 3. The time variation of: the integrated pressure force on the surface of the spheroid (blue filled squares); the integrated viscous force on the surface of the spheroid (red open circles); in the: $(\mathrm{a}, \mathrm{c}) x$-direction; and $(\mathrm{b}, \mathrm{d}) z$-direction averaged over the imposed vertical oscillation period $T$, for a locomotive flapping oblate spheroid at $R e_{A}=81$ with $A R=0.1$. The inset in (a) shows the time variation of the magnitude of the horizontal velocity (triangles) compared with a straight solid line on log-linear axes, demonstrating early time quasi-exponential growth consistent with a linear instability, while the dashed lines in (a) and (b) show the $x$-component and the $z$-component of the velocity respectively.

for an oblate spheroid with $A R=0.1$ at $R e_{A}=81$ in figure 2, well above the critical value for locomotion (a supplementary material movie is also available). Its horizontal trajectory is plotted in figure 1(a), with the magnified inset showing its initial trajectory in detail, and it is also marked with a circle symbol in figure 1(b)). As is now conventional, (Jeong \& Hussain 1995), the shed vortices are visualised using an appropriate positive value of the second invariant $Q$ of the velocity gradient tensor, i.e.

$$
Q=\frac{1}{2}\left[|\boldsymbol{\Omega}|^{2}-|\mathbf{S}|^{2}\right]>0,
$$

where $\mathbf{S}=\frac{1}{2}\left[\nabla \mathbf{v}+(\nabla \mathbf{v})^{T}\right]$ is the rate of strain tensor, and $\boldsymbol{\Omega}=\frac{1}{2}\left[\nabla \mathbf{v}-(\nabla \mathbf{v})^{T}\right]$ is the vorticity tensor. The positive value of $Q$ means that the Euclidean norm of the vorticity tensor dominates that of the rate of strain.

The flow loses axisymmetry at $t=4 T$, as two closely aligned vertical vortex structures connect two vortex rings shed at different cycles. At this stage, the horizontal velocity of the oblate spheroid grows quasi-exponentially in time as shown in the inset of figure 3 , consistent with the development of a linear instability. 'Older' vortex rings, i.e. those shed during previous vertical oscillation cycles, propagate away from the spheroid and grow as they entrain quiescent surrounding fluid (Maxworthy 1972). Later, further, in- 
herently nonlinear interactions between these shed vortices and the spheroid 'push' it into locomotion. As shown at $t=5 T$ in figure 2, the vortex rings tilt away from the spheroid due to perturbation growth. These tilted rings then induce imbalanced forces on the spheroid, causing it to accelerate towards a fully unidirectionally locomotive state, as shown at $t=10 \mathrm{~T}$ in figure 2 . At this asymptotic stage, the wake is dominated by two sets of vortex rings, shed at the previous oscillation cycle and well-known to be a propulsive wake (Dong et al. 2006).

The time-varying forces associated with this evolution leading to the asymptotic locomotion of the oblate spheroid are shown in figure 3. We plot separately the time evolution of the $x$-component (in figures $3(\mathrm{a})$ and (c)) and the $z$-component (in figures 3(b) and (d)) of the pressure force (i.e. the integral of the pressure over the surface of the oblate spheroid) with blue filled squares, and the time evolution of the viscous force (i.e. the integral of the viscous shear stress over the surface of the spheroid) with red open circles, averaged over the imposed vertical oscillation period $T$. We also plot (with dashed lines) the associated $x$-component (in figure $3(\mathrm{a})$ ) and the $z$-component (in figure $3(\mathrm{~b})$ ) of the induced velocity of the oblate spheroid.

It is not possible to decompose the forces acting on the spheroid into a pure propulsive force and a pure drag force. However, as is apparent in figure 3, when the spheroid exhibits unidirectional motion, the pressure forces act predominantly in the direction of motion, while the viscous forces predominantly act in the opposite direction, opposing the motion. Therefore, we think it is appropriate to consider the pressure force as a proxy for the propulsive force, and the viscous force as a proxy for the drag force. It is important to appreciate that this proxy is not precise, particularly right at the start of the locomotion, as can be seen in figure 3 , when both the viscous forces and the pressure forces act in the same direction.

We believe that this is due to the (slight) loss of up-down symmetry right at the initiation of locomotion. Although consideration of this particular stage of the flow evolution is beyond the scope of this study, we have checked that this brief initial period (when the viscous force contributes positively to propulsion) is robust with respect to variations in numerical resolution. We have repeated this numerical calculation with substantially enhanced mesh with over 3.5 million elements. We have particularly refined the mesh around the disk, with the height of the first cell layer being halved (and hence the number of cells on the disk surface being increased accordingly) with the time step also being halved so that the Courant-Friedrichs-Lewy requirement is still satisfied. The behaviour remains very similar, with the ultimate speed of propagation differing by less than $0.5 \%$ between the two simulations. The structure of the time-evolution of the viscous force is the same in both simulations, with a negative dip (acting in the direction of motion) at the onset of propagation rapidly changing to an asymptotic (positive) drag for both components.

However, there is some resolution-dependent variation in the timing and magnitude of this dip. For the simulation shown in figure 3 , the minimum $x$-component of the viscous force is -0.056 at $t / T=12$, and the minimum $z$-component of the viscous force is -0.045 at $t / T=6$, while for the simulation with enhanced resolution, the minimum $x$-component of the viscous force is -0.060 at $t / T=9$, and the minimum $z$-component of the viscous force is -0.130 at $t / T=9$. Nevertheless, we do not believe that these variations significantly alter the interpretation of this phenomenon as a physically meaningful earlytime property of this flow.

All the components of the forces on the spheroid unsurprisingly oscillate markedly and synchronously, i.e. with twice the frequency, conforming to similar structure on both the upstroke and downstroke of the spheroid's oscillation. Ultimately, when averaged 

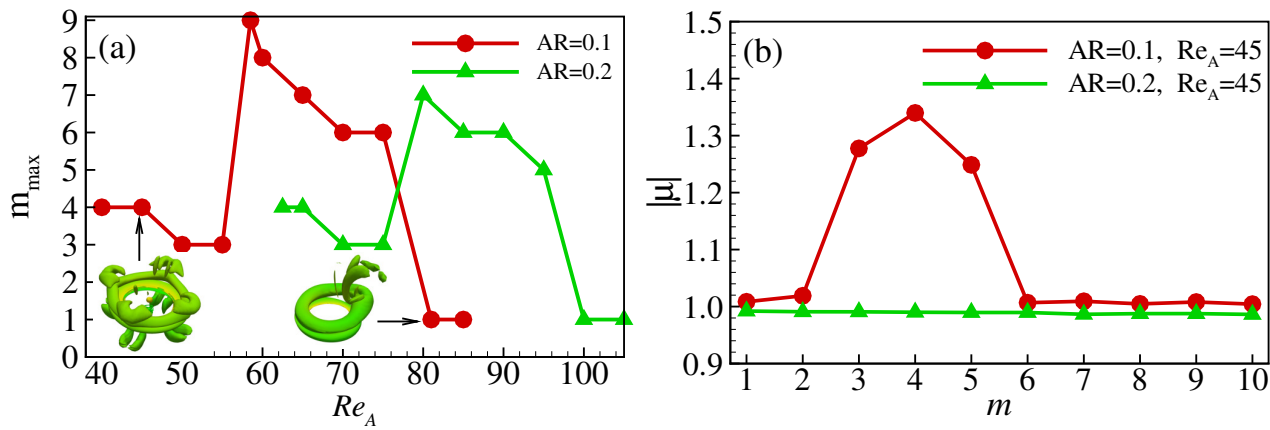

FiguRE 4. (a) Variation of the most unstable azimuthal wavenumber $m_{\max }$ with $R e_{A}$ for oscillating spheroids with $\beta=500$ and $A R=0.1$ (red circles) and $A R=0.2$ (green triangles). Instability onsets at $R e_{A} \simeq 40$ for $A R=0.1$, and at $R e_{A} \simeq 62$ for $A R=0.2$. The discontinuous jumps in wavenumber with increasing $R e_{A}$ are associated with transitions from branch ' $\mathrm{I}$ ' to branch 'O' to asymptotic $m=1$ instability (see Deng et al. (2016) and Deng et al. (2017) for further details). Inset are three-dimensional structures of the vorticity distributions, (visualized using $Q=50$ as defined in (3.1)) for: $R e_{A}=81$, showing the dominant $m=1$ asymmetric structure; and $R e_{A}=45$, showing the $m=4$ most unstable (branch 'I') mode from direct numerical simulations of horizontally fixed oscillating spheroids with $A R=0.1$. b) Variation of Floquet multiplier amplitude $|\mu|$ with azimuthal wavenumber $m$ for the flow around oscillating spheroids with $\beta=500, R e_{A}=45$ and $A R=0.1$ (red circles) and $A R=0.2$ (green triangles).

over a period, the forces are essentially in balance, leading to a constant locomotion velocity, with the pressure force driving and the viscous force resisting the motion as expected, consistent with the two-dimensional observations of Alben \& Shelley (2005). Furthermore, as shown on figure 1(a), there is a burst of $z$-component forces as the spheroid changes direction around $t / T_{0}=10$.

As is apparent from figure 2, and also in the accompanying supplementary material movie, unidirectional propulsion is associated with a characteristic vortex structure, with angled vortex dipoles effectively connecting the spheroid to both vortex rings which are periodically shed above and below the spheroid as it oscillates, an arrangement which is characteristic of a propulsive wake (Dong et al. 2006). This coupled vortex-structure may also be interpreted as the finite amplitude manifestation of the $m=1$ asymmetric linear instability of horizontally fixed oscillating spheroids identified in Deng et al. (2016) by Floquet stability analysis (cf. figure 2 of that paper). The initially quasi-exponential growth of the perturbation also naturally suggests that the transition to flying is associated with a linear instability, and there is clear structural similarity between the finite amplitude vorticity distribution around the flying ellipsoid and the eigenmode structure of the $m=1$ linear instability of the fixed spheroid, as shown inset in figure 4(a).

\subsection{Irregular locomotion at $A R=0.1, R e_{A}=45$ : 'Stair-step'}

As already noted, for flapping Reynolds numbers $R e_{A} \gtrsim R e_{C}$, the oblate spheroid exhibits complex locomotive states, analogously to the behaviour of two-dimensional flapping foils. As noted by Alben \& Shelley (2005); Lu \& Liao (2006), in flows with $R e_{A} \simeq R e_{C}$, the foil may move backwards and forwards with a period several times of the basic oscillation period, for reasons which are not as yet fully explained. For the spheroids which we are considering here, we observe two types of irregularly locomotive states for $R e_{A}=45$ for aspect ratios $A R=0.1$ and $A R=0.2$. This value of $R e_{A}$ appears to be close to the critical value $R e_{C}$ for locomotion, and significantly for both these aspect ratios there appears to be an intermediate range of $R e_{A}$ with non-zero $R e_{U}$ (and 
12

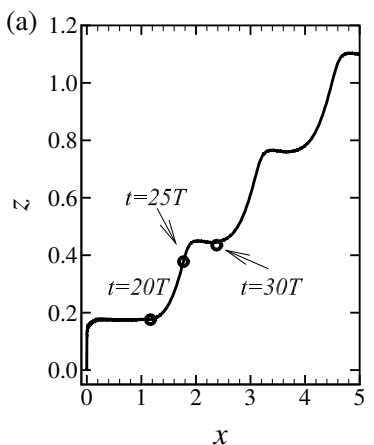

Jian Deng and C. P. Caulfield

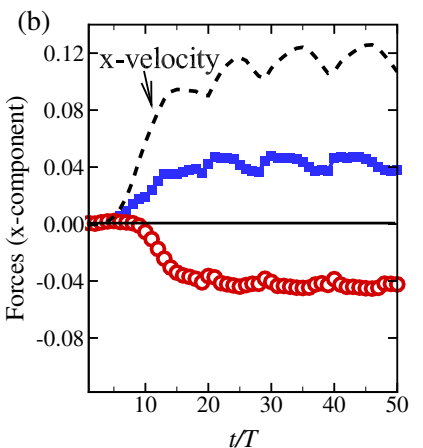

FIgURE 5. The dynamics of the irregularly locomotive oblate spheroid at $R e_{A}=45$ for $A R=0.1$. (a) The centre-of-mass trajectory projected to the horizontal $(x, z)$ plane. Note that: the locomotion starts from the origin; the total elapsed time shown is 50T; and the axes have different limits. The time variation of the: (b) $x$-components; and (c) $z$-components of horizontal pressure (blue filled squares), viscous forces (red open circles) and horizontal velocity (dashed lines) averaged over the imposed vertical oscillation period $T$. The vertical dashed lines in (c) correspond to the three times marked on (a).<smiles></smiles>
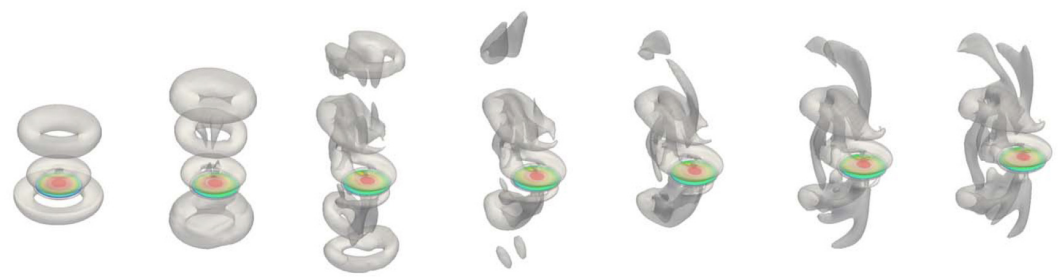$$
\mathrm{t} / \mathrm{T}=0
$$
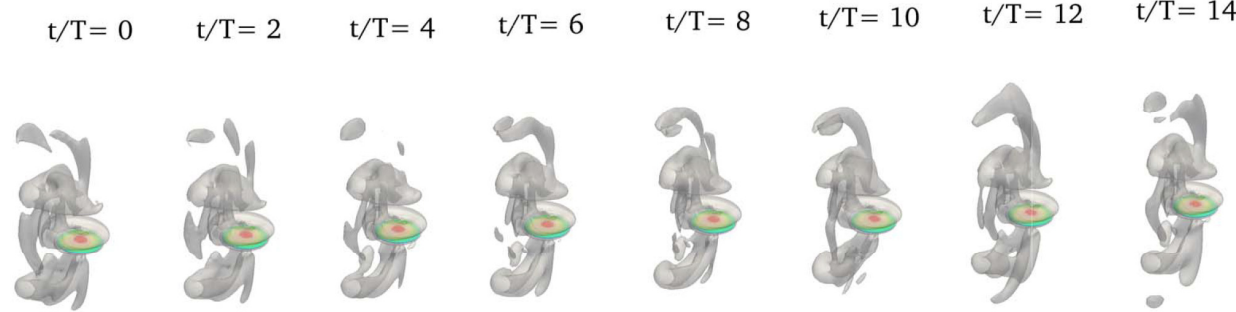

$$
\mathrm{t} / \mathrm{T}=16
$$

$$
\mathrm{t} / \mathrm{T}=20
$$$$
\mathrm{t} / \mathrm{T}=22
$$

$\mathrm{t} / \mathrm{T}=24$

$\mathrm{t} / \mathrm{T}=26$

$\mathrm{t} / \mathrm{T}=28$

$\mathrm{t} / \mathrm{T}=30$

FIGURE 6. Vortex topologies around the irregularly locomotive flapping oblate spheroid at $R e_{A}=45$ with $A R=0.1$, visualized using $Q=2$, as defined in (3.1). Images demonstrate flow dynamics associated with a direction change during irregular locomotion. The disk is coloured with the surface pressure distribution. A movie is also available as supplementary material.

thus 'flying') which still does not exhibit the asymptotic (close to constant) Strouhal number dependence.

As is apparent from figure $1(\mathrm{~b})$, increasing aspect ratio appears to reduce the extent in parameter space of the transition region to the asymptotic Strouhal number dependence, and thus we conjecture that the small aspect ratio spheroid has a wider irregular region. It is at least plausible that the higher wavenumber (i.e. with $m>1$ ) instabilities discussed in Deng et al. (2016) and Deng et al. (2017) occur strongly only when the spheroid is sufficiently 'thin', thus allowing excitation of sufficiently fine scale perturbations, although this has not been established by detailed analysis. 
The first type of irregularly locomotive state we have identified occurs for $R e_{A}=45$ and $A R=0.1$. This state takes the form of a 'stair-step' locomotion, various aspects of which are shown in figures 5 and 6 , and also in a movie available as supplementary material. All quantities oscillate synchronously with twice the frequency of the primary oscillation. The $x$-component of the velocity remains relatively constant about a nontrivial value, although there is also apparent a weaker, and slower frequency oscillation superposed. This 'slow' frequency oscillation (with period approximately 10T) is much more significant for the $z$-component of velocity, which drops to zero and then periodically spikes to much larger (and always positive in this coordinate system) values, briefly comparable with the $x$-component, leading to the characteristic 'stair-step' trajectory shown in figure 5(a) (note that the two axes have very different limits).

This behaviour (as well as the asymptotic steady locomotive state shown in figure 2) can be understood in terms of the spectral structure of the linear instabilities to which this flow is susceptible. We identify these instabilities using Floquet stability analysis, as described briefly in section 2.3. In figure 4 , we plot (with a solid line) the variation of the most unstable azimuthal wavenumber $m$ with $R e_{A}$ for a horizontally-fixed spheroid with $A R=0.1$, and Stokes number $\beta=500$. As discussed in more detail in Deng et al. (2016), there are three distinct branches of instability, which arise as $\operatorname{Re}_{A}$ (or equivalently $K C$ since $\beta$ is fixed) increases above $R e_{A}=40$. The first branch ' $\mathrm{I}$ ' is characterised by $m>1$ with in-phase perturbations above and below the oscillating disk, while the second branch ' $\mathrm{O}$ ' is typically at even higher $m>1$ with out-of phase perturbations. The switching of the dominant branch is associated with the discontinuous jump in the dominant wavenumber for $R e_{A} \simeq 60$, apparent in figure 4(a).

It appears that the irregular stair-step locomotive state at $R e_{A}=45$ can be interpreted in terms of the properties of these instabilities identified by Floquet analysis. Such an interpretation is particularly appealing if quasi-periodicity continues to be suppressed close to criticality in three-dimensional flow around a spheroid with $A R=0.1$ as we found occurs in two-dimensional flow (see Deng \& Caulfield (2016) for more details). As is shown in figure $4(\mathrm{a})$, at $R e_{A}=45$ the most unstable mode is predicted to be from branch 'I' with $m=4$. Crucially this imposes a four-fold symmetry on the most unstable mode, visualised by the inset vortical structure shown in figure 4 (a). The $m=4$ mode is not the only unstable mode however, as is shown in figure 4(b), as at these parameter values, both the $m=3$ and $m=5$ modes are predicted to be unstable, albeit with somewhat smaller growth rates.

Taken in combination, these various properties of the predicted linear instabilities (i.e. that the most unstable predicted mode has $m=4$ fourfold symmetry, and that modes with nearby wavenumbers also have nontrivial growth rates) suggest an appropriate argument for the observed stair-step propagation. As is apparent through comparison of figures 2 and 6 , the locomotive stair-step state has some similarities with unidirectional locomotion, in particular that it is characterised by a propulsive pair of vortices connecting the disk to the previously shed vortex rings. We conjecture that these structures develop from the interaction of neighbouring vortices arising from the $m=4$ instability through a symmetry-breaking bifurcation, thus preferring one 'side' of the 'square' (defined by the four-fold symmetry of potentially interacting vortices at the 'corners') of the most unstable eigenmode, leading to propagation in some preferred 'stair' direction. However, the most unstable $m=4$ mode is modulated by (the also growing) $m=3$ and $m=5$ modes, leading to a temporal variation of the relative strength of the perturbations localised at each of the corners, such that the alignment of the dominant 'side' (i.e. the dominant interacting neighbouring vortices) switches to a neighbouring side leading to propagation in the 'step' direction, at approximately ninety degrees to the 'stair' 
direction. This switching appears to continue indefinitely, with the timing of the switching being apparently determined by the interactions between the modes associated with different wavenumbers, reminiscent of a relatively low frequency 'beating' response.

This 'stair-step' behaviour appears to be robust to variations in resolution, as similar behaviour occurs when we use the same (finer) mesh and shorter time-steps for a high resolution simulation as we used to test the robustness of the viscous force 'dip' shown in figure 3. The trajectory exhibits the same stair-step structure, with similar mean period between steps for example. Furthermore, this observation that the behaviour close to transition to locomotion for free-to-fly three-dimensional bodies of small aspect ratio can be interpreted in terms of the Floquet stability properties of horizontally-fixed bodies is consistent with the dynamics of two-dimensional foils discussed in Deng \& Caulfield (2016). Specifically, we found that freedom to move horizontally for such foils both significantly encouraged the onset of asymmetry and also suppressed (completely) the onset of $Q P_{L}$-type quasi-periodicity. Therefore, it is perhaps unsurprising that we do not observe quasi-periodicity for such low aspect ratio flying ellipsoids, and also that the Floquet stability analysis associated with horizontally-fixed bodies is relevant to the interpretation of the motion of bodies free to move horizontally.

\subsection{Floquet stability analysis for unidirectional motion at $A R=0.1, R e_{A}=81$}

Indeed, returning to the unidirectional locomotion observed at higher $R e_{A}$ described in the previous subsection, the observed dynamics in that regime can also be interpreted in terms of Floquet stability analysis. As discussed in more detail in Deng et al. (2016), for horizontally-fixed oscillating spheroids, as $R e_{A}$ increases to sufficiently large values, only the amplitude of the oscillation is significant, and for

$$
R e_{H} \equiv \frac{4 \pi^{2} f_{0} A^{2}}{\nu}=\frac{\left(2 \pi R e_{A}\right)^{2}}{\beta}>467
$$

the flow is found to be susceptible to instabilities dominated by a distinct $m=1$ asymmetry, which since we have fixed $\beta=500$ in this study, corresponds to $R e_{A} \gtrsim 80$ (see figure 4).

This higher $R e_{A}$ regime essentially corresponds to a loss of coupling horizontally across the oscillating spheroid, and the nonlinear vortical structure associated with an instability in this regime at $R e_{A}=81$ for a fixed oscillating spheroid is also shown inset in figure 4(a). This structure is very reminiscent of the finite amplitude vortical structures shown in figure 2 for asymptotic unidirectional motion. Once again, it is useful to remember the two key pieces of evidence from our two-dimensional studies at this aspect ratio $A R=0.1$, (see Deng \& Caulfield (2016) for more details) to interpret the data presented in these two figures: that being free to move horizontally reduces the critical flapping Reynolds number at which symmetry-breaking occurs; and also being free to move horizontally suppresses the occurrence of $Q P_{L}$-type quasi-periodicity (for low aspect ratio bodies) close to criticality. Therefore, by analogy, in three dimensions we conjecture that the range of flapping Reynolds numbers susceptible to complex, higher wavenumber instabilities is reduced when the body is free to move horizontally. This is a possible explanation for why asymptotic unidirectional motion is observed for $R e_{A}=63$ for example, although the linear Floquet analysis as shown in figure 4(a) (crucially for horizontally-fixed spheroids) predicts a higher wavenumber $\left(m_{\max }=7-8\right)$ primary instability at this value of flapping Reynolds number. 
(a)

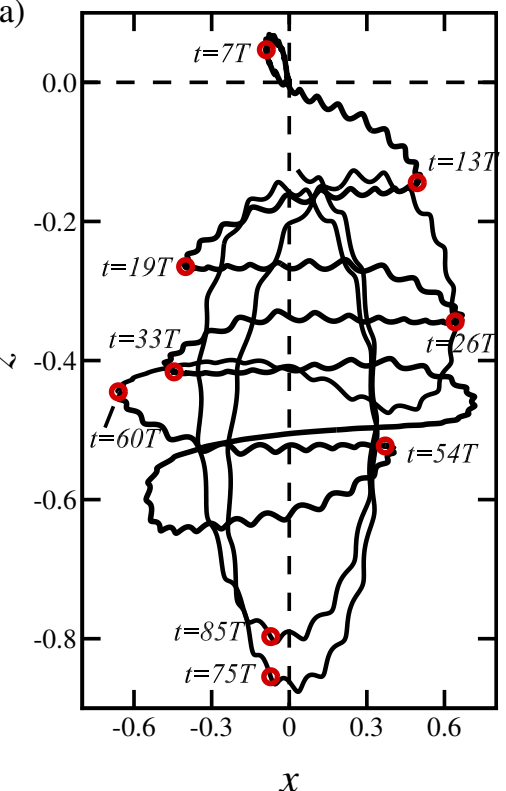

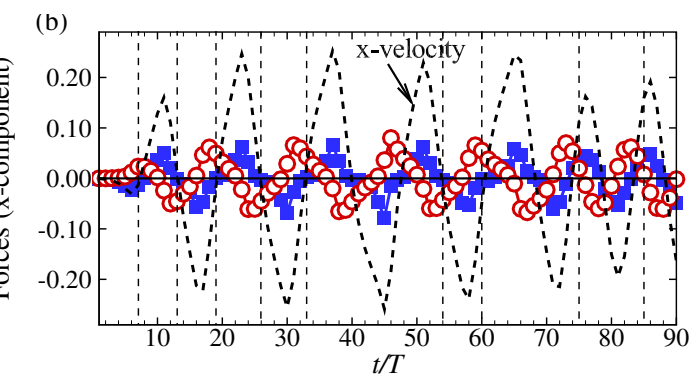

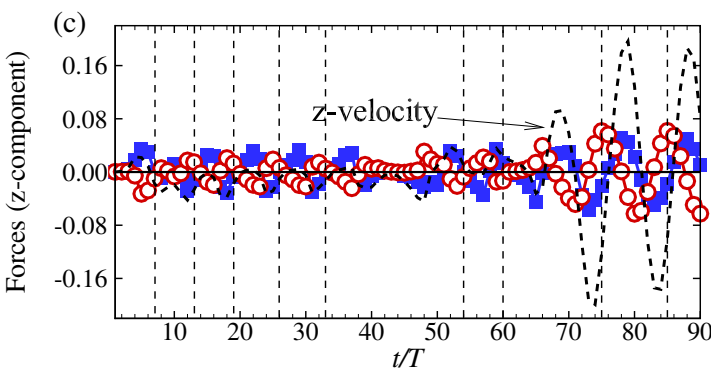

FIGURE 7. The dynamics of the irregularly locomotive oblate spheroid at $R e_{A}=45$ for $A R=0.2$, with $m_{s} / m_{f}=10$. (a) The centre-of-mass trajectory projected to the horizontal $(x, z)$ plane. Note that: the locomotion starts from the origin; the total elapsed time shown is $90 T$; and the axes have different limits. The time variation of the: (b) $x$-components; and (c) $z$-components of horizontal pressure (blue filled squares), viscous forces (red open circles); and horizontal velocity (dashed lines) averaged over the imposed vertical oscillation period $T$. The dashed vertical lines in (b) correspond to the times marked on (a).
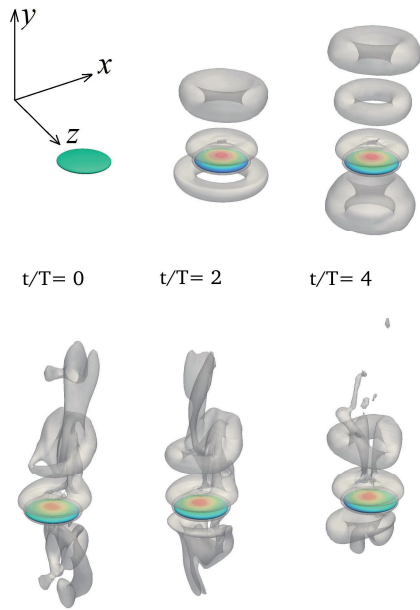

$t / T=18$ $\mathrm{t} / \mathrm{T}=2$

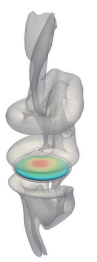

$\mathrm{t} / \mathrm{T}=20$ $\mathrm{t} / \mathrm{T}=4$

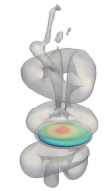

$\mathrm{t} / \mathrm{T}=22$
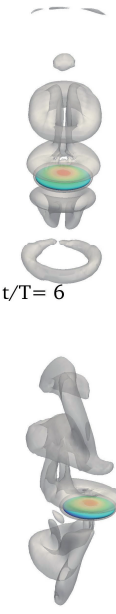

$\mathrm{t} / \mathrm{T}=24$
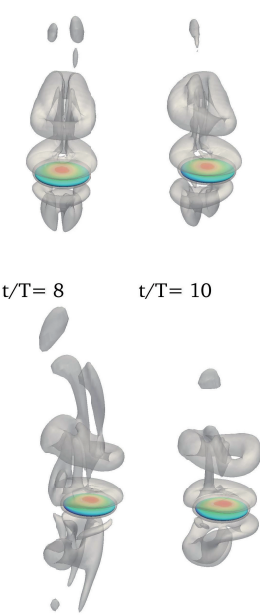

$\mathrm{t} / \mathrm{T}=26$

$\mathrm{t} / \mathrm{T}=10$

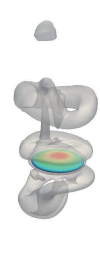

$\mathrm{t} / \mathrm{T}=28$
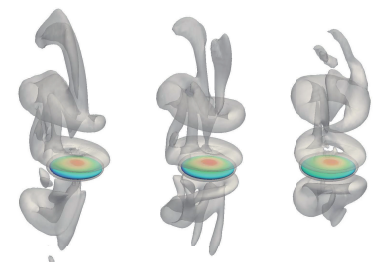

$\mathrm{t} / \mathrm{T}=12$

$\mathrm{t} / \mathrm{T}=16$
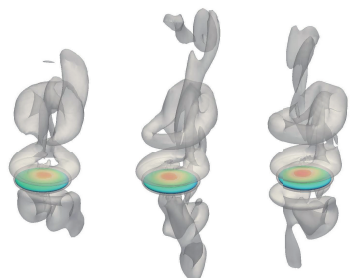

$\mathrm{t} / \mathrm{T}=32$

$\mathrm{t} / \mathrm{T}=34$

Figure 8. Vortex topologies around the irregularly locomotive flapping oblate spheroid at $\operatorname{Re}_{A}=45$ with $A R=0.2$, visualized using $Q=2$, as defined in (3.1). Images demonstrate flow dynamics associated with a direction change during the relatively early 'snaking' irregular locomotion. The disk is coloured with the surface pressure distribution. A movie is also available as supplementary material. 


\subsection{Irregular locomotion at $A R=0.2, R e_{A}=45$ : 'Snaking'}

The second type of irregular locomotion, which we refer to as 'snaking', occurs at early times for $R e_{A}=45$ for an oblate spheroid with $A R=0.2$. Certain properties of this 'snaking' flow are shown in figures 7 and 8. Although the stresses and the velocities once again oscillate at twice the primary frequency, it is appropriate to describe the dynamics here as quasi-periodic, as the $x$-component of velocity exhibits large sawtooth variation between roughly comparable positive maximum and negative minimum values, with the $z$-component exhibiting (in general different) amplitude oscillations of both signs (though initially with non-zero negative mean in this coordinate system) when the $x$-component is close to its maximum amplitude. Such coupled oscillations lead initially to the long, sweeping quasi-periodic downward 'snaking' trajectory shown for early times in figure $7(\mathrm{a})$. In figure 8, the direction switching phenomena for the early-time 'snaking' locomotion is clearly observed.

Ultimately, as is apparent in figure 7, the 'snaking' trajectory locks into a close to elliptical 'orbit' and the spheroid undertakes quasi-periodic motions around this orbit. As is apparent in the movie available as supplementary material, the vortical structures around the spheroid are very similar during the 'snaking' and 'orbiting' phases of the flow evolution. Furthermore, this 'snaking' leading to 'orbiting' behaviour is also robust to variation in resolution, as qualitatively very similar dynamics arise when the flow is simulated with the finer mesh used to test the early-time 'dip' in the viscous force (shown in figure 3) and the 'stair-step' locomotion for smaller aspect ratio ellipsoids shown in figure 5 .

This locomotion behaviour can also be interpreted in the light of our previous observation (see Deng \& Caulfield (2016)) that in two dimensions, the initial symmetry-breaking for such higher aspect ratio bodies is expected to be quasi-periodic in character, as being free to move horizontally actually encourages the appearance of the $Q P_{H}$-type asymmetry, analogously to the behaviour observed here. Indeed, this scenario is further reinforced by a careful consideration of the results of our Floquet stability analysis for a flow around a horizontally fixed spheroid with these parameter values. As shown in figure 4(b), for a fixed spheroid, a flow with this particular value of $R e_{A}$ is actually predicted to be stable, as the range of Reynolds numbers susceptible to branch ' $\mathrm{I}$ ' and branch ' $\mathrm{O}$ ' instabilities shifts to higher Reynolds number as the aspect ratio increases.

As already noted above, it is a reasonable hypothesis that the critical value of $R e_{A}$ for symmetry breaking may be somewhat smaller for spheroids which are free to 'fly' compared to spheroids which are fixed horizontally, by analogy with inherently twodimensional flows. Nevertheless, as shown in figure 4(a), the critical Reynolds number for the onset of instability (also with $m=4$ at this aspect ratio) is $R e_{A} \simeq 62$. Therefore, it seems unlikely that the flow at the substantially smaller $R e_{A}=45$ is still linearly unstable, even when the spheroid is not fixed horizontally.

There are two further reasons why we do not believe that this irregular locomotion is related to a modal flow instability. First, the evolution is inherently quasi-periodic in character, with an apparent competition between several characteristic angles of direction change and periods of flow evolution. Second, particularly for this irregularly locomotive state, viscous forces clearly play a non-trivial and complex role. As can be seen in figures 7 (b) and (c), when averaged over a cycle of the vertical oscillation, as expected pressure forces act predominantly in the same direction as the motion, while the viscous forces act in the opposite direction. However, these correlations are by no means perfect, and it is apparent that there are time intervals where the viscous forces actually act in the same direction as the motion. Using the root-mean-square $u_{r m s}$ of the locomotive velocity 
(a)

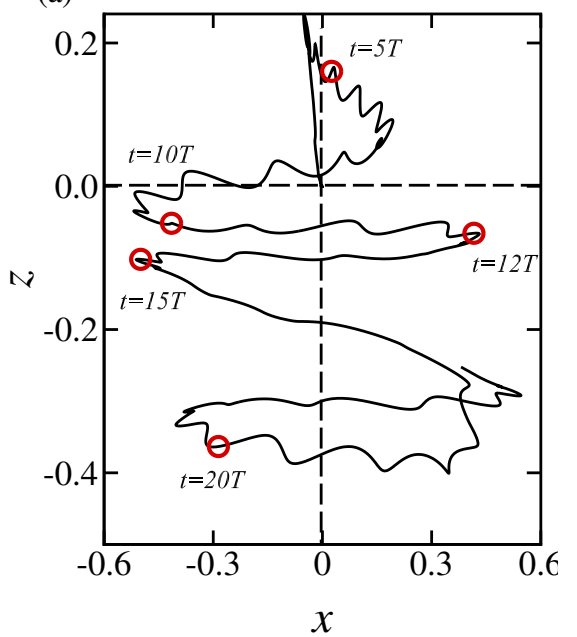

(b)

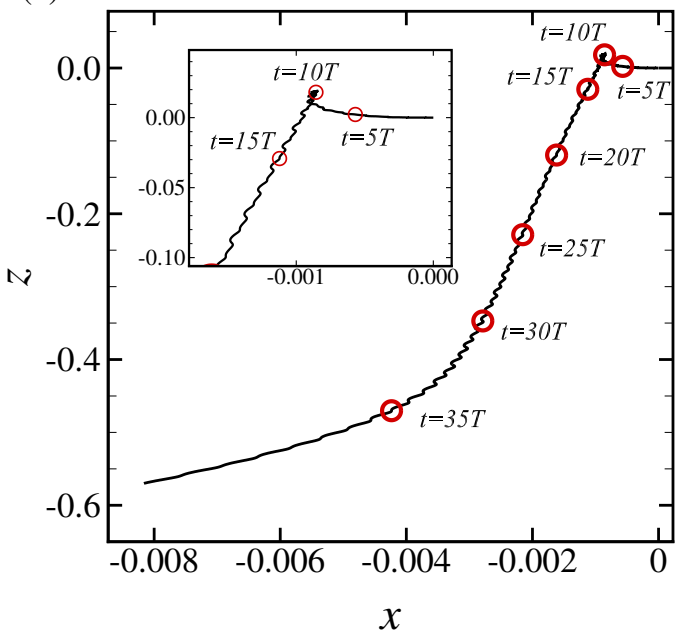

FiguRE 9. The centre-of-mass trajectory projected to the horizontal $(x, z)$ plane for an oblate spheroid with $R e_{A}=45$ and $A R=0.2$ with mass ratio: (a) $m_{s} / m_{f}=1$; and (b) $m_{s} / m_{f}=100$.

to define the characteristic velocity scale $U$ in the definition (2.1) for the locomotion Reynolds number $R e_{U}$ for this relatively slow 'snaking' locomotion leads to $R e_{U} \simeq 10$. Since the aspect ratio is $A R=0.2$, an appropriate Reynolds number for this motion using the vertical rather than horizontal dimension of the oblate spheroid $R e_{V}=u_{r m s} b / \nu \sim$ $\mathcal{O}(1)$, suggesting that Stokes effects may play a major role, in particular leading to intermittent positive viscous contributions to the locomotion.

Major viscous effects may also be the dominant cause of the changes of direction, associated with complex vortex ring shedding processes. Similarly to the unidirectional flows at higher $R e_{A}$, the main mechanism for forward propulsion is associated with close to vertical vortex dipoles on either side of the spheroid. At this lower value of $R e_{A}$ however, these vertical vortices inevitably diffuse and 'fatten' more quickly, and remain more closely connected to the vortex rings in the immediate vicinity of the spheroid. This is particularly apparent in the images for $t=18 T$ and $t=20 T$ in figure 8 , at instants either side of the turn at $t=19 T$, as marked on figure 7(a). This instant is associated with a peak in the magnitude of viscous forces (see figures $7(b)$ and (c)) as these close to vertical vortices disrupt asymmetrically the shedding of the next vortex ring, thus apparently triggering a marked change in the direction of propagation.

This clearly strongly nonlinear dynamical behaviour further suggests that this irregular 'snaking' locomotion state should be thought of as being inherently quasi-periodic, and a natural three-dimensional generalization of the $Q P_{H}$-type asymmetry previously reported for two-dimensional foils particularly as the coupling between these shed vortices eventually locks the spheroid into an elliptical orbital motion which does not appear to lead to sustained (unidirectional) locomotion. This nonlinear, yet still spatially bounded, ultimate response strongly suggests that the dynamical behaviour should not be interpreted as being driven by a (linear) flow instability, though detailed investigation of the (apparent) basin of attraction of this snaking to orbiting state is beyond the scope of this study. 


\subsection{Mass ratio effects on 'snaking' at $A R=0.2, R e_{A}=45$}

Throughout the rest of this study, we have considered oscillating spheroids with a mass ratio $m_{s} / m_{f}=10$. It is important to appreciate that this ratio can play an important role in the dynamics of a flapping oblate spheroid, although the onset Reynolds numbers are independent of this ratio, as discussed by Alben \& Shelley (2005). For completeness, we present in figure 9 the centre-of-mass trajectories for oscillating spheroids with $R e_{A}=45$ and $A R=0.2$ (i.e. the same parameters as the spheroid with properties shown in figure 7) with two different mass ratios: $m_{s} / m_{f}=1$; and $m_{s} / m_{f}=100$.

The low mass ratio $\left(m_{s} / m_{f}=1\right)$ spheroid, as shown in figure $9(\mathrm{a})$, exhibits even more irregular motion than the spheroid with $m_{s} / m_{f}=10$, although the low mass ratio spheroid still exhibits a qualitatively similar 'snaking' trajectory which is just entering an ultimate 'orbit'. On the other hand, the high mass ratio $\left(m_{s} / m_{f}=100\right)$ spheroid, as shown in figure $9(\mathrm{~b})$, eventually settles into a unidirectional locomotive state. Nevertheless, there is still an initial period of irregular motion, which also has a slight 'judder' with the same period as the vertical oscillation, reminiscent of the trajectory for the spheroid with $m_{s} / m_{f}=10$ shown in figure 7 . Such suppression of irregular motion by increasing mass ratio is interesting, though perhaps not entirely surprising. We do not investigate this issue further here, as it is not central to the aims of this particular study.

\section{Conclusions}

We have considered numerically the locomotion of vertically oscillating or flapping oblate spheroids for a range of flapping Reynolds numbers $R e_{A}$ and aspect ratios. We can draw three main conclusions. First, at sufficiently large flapping Reynolds numbers $R e_{A}$ as defined in (1.1), unidirectional locomotion emerges as an attracting state for a vertically-oscillating spheroid which is free to move horizontally. Second, that locomotion is somewhat less efficient (in the sense of having higher Strouhal number $S t$ as defined in (1.1)) than in the previously considered strictly two-dimensional flow and it onsets at appreciably higher amplitudes of oscillation (or equivalently at higher critical values of $\left.R e_{A}\right)$. We also find that the efficiency actually decreases with increasing aspect ratio, but in a way such that the relative inefficiency compared to two-dimensional motion becomes less significant. And third, before the emergence of such unidirectional locomotion, (i.e. for $R e_{A}$ only slightly larger than its critical value $R e_{C}$ ) irregular locomotion can be observed, which can be usefully interpreted in terms of the Floquet stability properties of horizontally-fixed oscillating spheroids. In particular, there is at least some evidence that when $R e_{A} \gtrsim R e_{C}$ for low aspect ratio spheroids, the 'in phase' branch 'I' of high azimuthal wavenumbers predicted in Deng et al. (2016) and experimentally observed in Deng et al. (2017) can trigger a 'stair-step' locomotion, while for higher aspect ratio spheroids, an essentially quasi-periodic 'snaking' locomotion can occur at early times, which then leads to an ultimate bounded elliptical 'orbit'. We conjecture that this quasiperiodic behaviour is a three-dimensional generalization of the ' $Q P_{H}$ '-type quasi-periodic symmetry breaking previously identified in inherently two-dimensional flows in Deng \& Caulfield (2016). We find that this quasi-periodic behaviour is not explainable in terms of a linear instability identified using Floquet stability analysis of a horizontally-fixed spheroid, and also that it is suppressed by increasing the mass ratio of the spheroid compared to the fluid.

Unsurprisingly perhaps, there is a much richer range of locomotion possible for such inherently three-dimensional spheroids than for equivalent two-dimensional foils, not least because the induced vortex-ring flows can exhibit such complex dynamics. Indeed, al- 
though vertical flapping can still lead to unidirectional horizontal locomotion for small aspect ratio three-dimensional oblate spheroids, and there are undoubtedly some phenomena which are analogous to those observed in two dimensions, application of insights gained from strictly two-dimensional modelling to more realistic three-dimensional flows should be tested carefully. Of particular interest is to investigate in detail the properties of the inherently nonlinear quasi-periodic 'snaking' to 'orbiting' state identified for sufficiently large aspect ratio oscillating ellipsoids. Clearly, the vortices shed during the various periods couple in some way to lead to a bound, yet non-stationary state, with a particularly complex interaction occuring between the close to vertical 'steering' vortex pairs and the subsequently shed vortex rings (as is visible in figure 8 and the associated movie available as supplementary material). Furthermore, for the spheroids considered here, viscous stresses clearly play a central role, (not least right at the beginning of the onset of locomotion when the 'dip' in viscous forces as shown in figure 3 actually contributes constructively to forward propulsion initially) and while the flows do appear to exhibit nontrivial instabilities, the shed vortex rings themselves do not break down vigorously, but rather decay viscously as the spheroid oscillates and propagates horizontally.

It is clearly of interest to extend the considered parameter range to investigate the behaviour at higher Reynolds number where the wake of the spheroid becomes turbulent. Obvious objectives would be to identify the range over which the observed efficiency of locomotion survives, as well as to explore the stability of the oscillating spheroids if they in turn are allowed to pitch and roll in response to the induced fluid motion, i.e. to determine whether or not they are unstable to infinitesimal rotations about their principal axes of inertia. The feedback between the induced vortical motions in the 'wake' and the oscillatory motion of a fully freely moving spheroid has the undoubted potential to lead to an even richer range of dynamically interesting motions, possibly connected to the well-known complex motions of freely falling bodies such as coins in water or leaves in the air (see Ern et al. (2012) for a recent review).

J. Deng has been supported by the National Natural Science Foundation of China (Grant No. 11772299), the State Key program of National Natural Science Foundation of China (Grant No: 11332009) to conduct this research. J. Deng gratefully acknowledges the hospitality of the Department of Applied Mathematics \& Theoretical Physics, University of Cambridge, and the award of a by-fellowship by Churchill College, University of Cambridge. Thanks are also due to four anonymous reviewers, whose thoughtful and constructive comments have led to a substantial improvement in the robustness and clarity of the presentation of the results in this paper.

\section{REFERENCES}

Alben, S. \& Shelley, M. 2005 Coherent locomotion as an attracting state for a free flapping body. Proc. Natl Acad. Sci. USA 102, 11163-11166.

Alexander, R. M. 1993 Principles of animal locomotion. Princeton University Press.

BAR-Cohen, Y. 2006 Biomimetics: Using nature to inspire human innovation. Bioinspiration \& biomimetics 1 (1), P1.

Childress, S. 1981 Mechanics of swimming and flying. Cambridge University Press.

Childress, S. \& Dudley, R. 2004 Transition from ciliary to flapping mode in a swimming mollusc: flapping flight as a bifurcation in $R e_{\omega}$. J. Fluid Mech. 498, 257-288.

Deng, J. \& Caulfield, C. P. 2016 Dependence on aspect ratio of symmetry breaking of oscillating foils: implications for flapping flight. J. Fluid Mech. 787, 16-49.

Deng, J., Teng, L. B., Caulfield, C. P. \& MaO, X. R. 2016 Instabilities of interacting vortex rings generated by an oscillating disk. Phys. Rev. E. 94, 033107. 
Deng, J., Xue, J. Y., MaO, X. R. \& Caulfield, C. P. 2017 Coherent structures in interacting vortex rings. Phys. Rev. Fluids 2, 022701.

Dong, H., Mittal, R. \& Najiar, F. M. 2006 Wake topology and hydrodynamic performance of low-aspect-ratio flapping foils. J. Fluid Mech. 566, 309-343.

Dreyfus, R., Baudry, J., Roper, M. L., Fermigier, M., Stone, H. A. \& Bibette, J. 2005 Microscopic artificial swimmers. Nature 437 (7060), 862-865.

Elston, J. R., Sheridan, J. \& Blackburn, H. M. 2001 The transition to three-dimensionality in the flow produced by an oscillating circular cylinder. In A/Asian Fluid Mech. Conf., pp. 319-322. Adelaide, Australia.

Ern, P., Risso, F., Fabre, D. \& Magnaudet, J. 2012 Wake-induced oscillatory paths of bodies freely rising or falling in fluids. Annu. Rev. Fluid Mech. 44, 97-121.

Ferziger, J. H. \& Peric, M. 2002 Computational methods for fluid dynamics. Spring, Berlin.

Fish, F. E., Lauder, G. V., Mittal, R., Techet, A. H., Triantafyllou, M. S., Walker, J. A. \& WeBb, P. W. 2003 Conceptual design for the construction of a biorobotic auv based on biological hydrodynamics. In Proc. 13th Intl Symp. on Unmanned Untethered Submersible Technology (UUST), pp. 1-8. New Hampshire, USA.

JASAK, H. 1996 Error analysis and estimation in the finite volume method with applications of fluid flows. Ph.D Thesis, Imperial College, University of London.

JeOng, J. \& Hussain, F. 1995 On the identification of a vortex. J. Fluid Mech. 285, 69-94.

Karniadakis, G. \& Sherwin, S. 2013 Spectral/hp element methods for computational fluid dynamics. Oxford University Press.

Klotsa, D., Baldwin, K. A., Hill, R. J. A., Bowley, R. M. \& Swift, M. R. 2015 Propulsion of a two-sphere swimmer. Phys. Rev. Lett. 115 (24), 248102.

Lewin, G. C. \& HAJ-HARIRI, H. 2003 Modelling thrust generation of a two-dimensional heaving airfoil in a viscous flow. J. Fluid Mech. 492, 339-362.

Lu, X. \& LiaO, Q. 2006 Dynamic responses of a two-dimensional flapping foil motion. Phys. Fluids 18, 098104.

Maxworthy, T. 1972 The structure and stability of vortex rings. J. Fluid Mech. 51, 15-32.

Tatsuno, M. \& Bearman, P. W. 1990 A visual study of the flow around an oscillating circular cylinder at low Keulegan-Carpenter numbers and low Stokes numbers. J. Fluid Mech. 211, $157-182$.

Taylor, G., Nudds, R. \& Thomas, A. 2003 Flying and swimming animals cruise at a Strouhal number tuned for high power efficiency. Nature 425, 707-711.

Techet, A. H., Lim, K. L., Hover, F. S. \& Triantafyllou, M. S. 2004 Hydrodynamic performance of a biologically inspired 3D flapping foil. In Proc. 14th Intl Symp. on Unmanned Untethered Submersible Technology (UUST). New Hampshire, USA.

Triantafyllou, G., Triantafyllou, M. \& Grosenbaugh, M. 1993 Optimal thrust development in oscillating foils with application to fish propulsion. J. Fluid. Struct. 7, 205-224.

Triantafyllou, M. S., Techet, A. H. \& Hover, F. S. 2004 Review of experimental work in biomimetic foils. IEEE J. Oceanic Engng 29, 585-595.

Vandenberghe, N., Childress, S. \& Zhang, J. 2006 On unidirectional flight of a free flapping wing. Phys. Fluids 18, 014102.

Vandenberghe, N., Zhang, J. \& Childress, S. 2004 Symmetry breaking leads to forward flapping flight. J. Fluid Mech. 506, 147-155.

Williams, B. J., Anand, S. V., Rajagopalan, J. \& Saif, M. T. A. 2014 A self-propelled biohybrid swimmer at low Reynolds number. Nature Comm. 5, Art. No. 3081.

Zhang, X., Ni, S., Wang, S. \& He, G. 2009 Effects of geometric shape on the hydrodynamics of a self-propelled flapping foil. Phys. Fluids 21, 103302. 